Review article

\title{
Traumatic spinal cord injuries
}

\author{
Naveen Kumar ${ }^{\mathrm{a}, *}$, Aheed Osman ${ }^{\mathrm{a}}$, J.R. Chowdhury ${ }^{\mathrm{b}}$ \\ ${ }^{a}$ Consultant Surgeon in Spinal Injuries, Midland Centre for Spinal Injuries, RJAH Orthopaedic Hospital NHS Foundation Trust, Oswestry, Shropshire SY10 7AG, \\ UK \\ ${ }^{\mathrm{b}}$ Clinical Lead \& Consultant Surgeon in Spinal Injuries, Midland Centre for Spinal Injuries, RJAH Orthopaedic Hospital NHS Foundation Trust, Oswestry, \\ Shropshire SY10 7AG, UK
}

\section{A R T I C L E I N F O}

\section{Article history:}

Received 9 May 2017

Received in revised form 7 June 2017

Accepted 10 June 2017

\section{Introduction}

The management of the traumatic spinal injury has remained controversial since the times of Charles Bell and Astley Cooper. Traumatic spinal injuries in 1920s and 1930s were non-operatively managed. Methods of reduction included hyperextension in the prone position on slings, frames or hammock, as described by Davis and Rogers or hanging (Bohler). Watson-Jones used the two-table method in 1931 and 1934. Dunlop and Parker hyper extended the broken spine in supine position. Magnus renounced methods of forceful reduction and accepted the spinal deformity. He advocated allowing the fractured spine to consolidate by placing the patient flat in bed in supine position with prolonged recumbency for 3-6 months or longer. Such prolonged immobilisation was carried out with often poor attention and poor management of the associated multisystem physiological impairment and malfunction. These methods of management have been strongly condemned (Guttmann, Watson-Jones, Holdsworth and Hardy) as utterly contrary to the principles of rehabilitation of spinal cord injured patients.

\section{Spinal cord injury service}

In England, on February 1, 1944, Sir Ludwig Guttmann established a spinal unit at Stoke Mandeville, UK and introduced multidisciplinary staffing for the comprehensive treatment and rehabilitation of SCI. Under his leadership, this unit became a world-renowned centre for teaching, research, and clinical care.

\footnotetext{
* Corresponding author.

E-mail addresses: naveen.kumar@rjah.nhs.uk (N. Kumar), aheed.osman@rjah.nhs.uk (A. Osman), joy.chowdhury@rjah.nhs.uk (J.R. Chowdhury).
}

Guttmann introduced and developed the method of graduated reduction of fractures and fracture dislocations of the injured spine and immobilisation on pillow packs while providing simultaneous detailed attention to the multisystem malfunction together with all medical and non-medical effects of paralysis.

The spinal cord injury ( $\mathrm{SCI}$ ) service in the UK from that time started to improve and increased the number of specialised centres for SCI throughout the country with specialised SCI healthcare services currently provided in eight specialist centres in England, and one each in Wales, Scotland and Northern Ireland, offering support for patients sustaining SCI through the initial period of treatment and rehabilitation and on-going lifelong support. Each of these centres has a specified geographical area's population to cover. The Midland Centre for Spinal Injuries (MCSI) is a part of the famous The Robert Jones and Agnes Hunt Orthopaedic Hospital, at Oswestry. Shropshire and was set up in 1965 by Mr T McSweeny and Dr B. F. Jones. MCSI covers around a 10 million population (Fig. 1).

Sir Ludwig Guttmann demonstrated that almost all of the complications that were believed to be inevitable following a $\mathrm{SCI}$ were indeed preventable. He asserted that complications following $\mathrm{SCI}$ are attributable to poor management of the patient rather than the neurological impairment or the patient being treated with bed rest. Interestingly although anatomical alignment was rarely achieved, Guttmann demonstrated that with simultaneous attention to all medical and non-medical effects of the SCI a significant number of patients recovered motor and sensory functions to ambulate and the majority were pain free following conservative management.

Based on such evidence the Active Physiological Conservative Management (APCM) of the spinal injury and its effects was described and popularised by Wagih El Masri pupil of Guttmann.

Wagih El Masri demonstrated that with expert, early, simultaneous APCM of the injured spine, spinal cord and all the medical and non-medical effects over $70 \%$ of patients with complete motor paralysis but with sparing of pin prick sensation presenting in the first $72 \mathrm{~h}$ of injury recover motor power to ambulate without surgical, pharmacological, cellular or biological intervention. Those presenting within $72 \mathrm{~h}$ of injury with motor sparing, however minimal the sparing is have an even better chance to walk, also without any intervention. El Masri et al. also demonstrated that the reduction of the period of treatment in recumbence from 12 weeks 


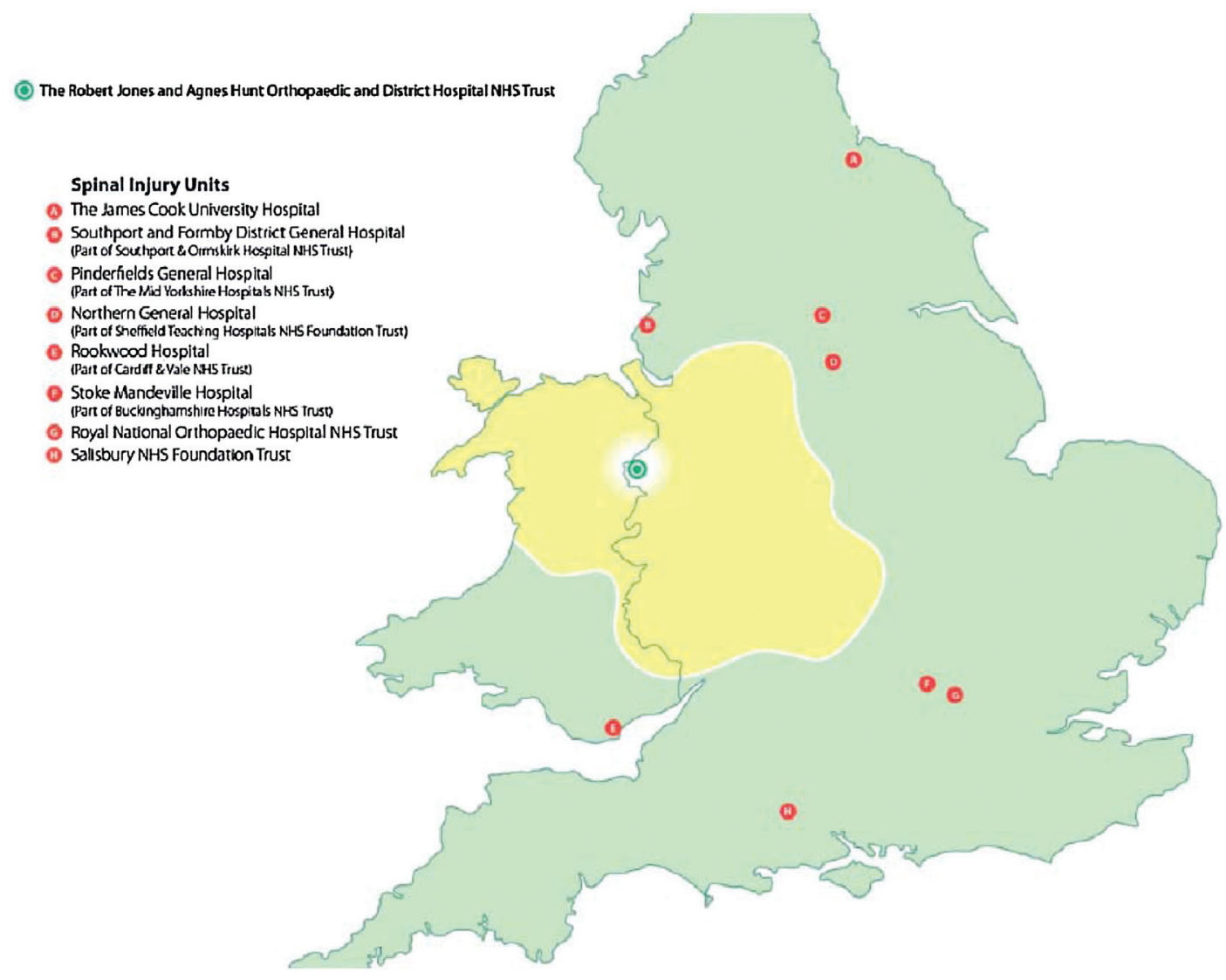

Fig. 1. Location of the UK spinal injury centres. The catchment area for the Midland Centre for Spinal Injuries is shown in yellow.

to between 4 and 6 weeks was safe both in the short and long term. They also demonstrated that with APCM the impact on the patient and family members can be minimised in both the short and long term. Patients who do not recover ambulation can with APCM and ongoing expert monitoring, care and support lead dignified, healthy, fulfilling, productive and often competitive lives.

Active Physiological Conservative Management, from the early hours of injury requires simultaneous scrupulous care of: the injured spine, the multisystem neurogenic effects of the spinal cord injury on the respiratory, cardiovascular, urinary, gastrointestinal, dermatological, sexual and reproductive functions, the management of the associated psychological effects of paralysis, the physical rehabilitation and the modification of the environment. By definition APCM requires between 4 and 6 weeks of treatment in recumbence. This is in order to rest injured tissue, prevent significant postural hypotension or significant reduction of vital capacity during the stage of spinal shock, minimise the risk of ischial and sacral pressure sores during the vulnerable period of poor skin perfusion due to spinal shock, facilitate intermittent catheterisation, facilitate bowel care and facilitate nursing care during the first few weeks of paralysis. It also allows for some recovery of the sympathetic nervous system reflexes which are paramount for the active cooperation of the patient with the demands of physical rehabilitation.

\section{Care of the injured spine - vertebral column stabilisation}

Although almost every patient in our institution is given informed choice between conservative and surgical management the majority of patients with SCI chose APCM. APCM method of management begins with active early vertebral column stabilisation. Most injuries of the vertebral column do not result in spinal cord damage, but traumatic spinal instability places the patient at risk for neurologic injury if the injured spinal segments are not protected. All trauma victims, especially those complaining of neck or back pain, are properly immobilised as quickly as possible. The most commonly used devices are adequate for emergency use but may not be appropriate for long-term stabilisation. Because of this, alternate forms of spinal immobilisation for patients judged to have marked spinal instability are employed soon after intensive care unit admission.

Patients with traumatic spinal injuries are kept supine and carefully moved, using a log-rolling technique to avoid flexion or extension of the spine. They are immobilised in standard beds, rotating beds, or turning frames. Turning frames require considerable nursing expertise for safe and effective use. The use of turning frames by inexperienced personnel is likely to result in inadequate spinal stabilisation.

Guttmann introduced, with the co-operation of Egerton Engineering Ltd, an electrically-controlled turning and tilting bed, which proved very satisfactory in reducing the broken spine and preventing sores not only in traumatic paraplegics but, by adding a special head traction unit, also in tetraplegics. Since $1960 \mathrm{~s}$ this is specialised turning and tilting bed has been widely utilised in managing victims with injuries of the vertebral column at Oswestry.

The facts however are those in recumbence and with some careful handling of the patient, neurological deterioration is very rare even in the most biomechanically unstable injuries. Similarly vertebral misalignment, canal encroachment and cord compression do not prevent neurological recovery and are rarely, individually or in combination the cause of neurological deterioration when patients are adequately managed with APCM. ${ }^{24-30}$ 
What is often overlooked is that the injured spinal cord is "Physiologically Unstable" because of the cellular and cell membrane disturbances, loss of auto-regulatory functions and disruption of blood brain barrier. ${ }^{19}$ The physiologically unstable injured spinal cord cannot protect itself from non-mechanical complications outside the spinal canal such as hypoxia, hypotension, hypertension, sepsis and hypothermia. These complications rarely cause paralysis in the neurologically intact patients. In a patient with spinal cord injury and a physiologically unstable spinal cord these complications can however be at least as damaging to the injured neural tissues as the potential mechanical damage caused by the mismanagement of the biomechanical instability of the injured column.

\section{Instability}

The diagnosis of biomechanical instability is usually based on radiological investigations at the time of the presentation of the patient. Clinically and radiologically any osseous fractures of any component of the vertebral unit of motion will heal within 6-12 weeks from injury. Restoration of the biomechanical stability of the spinal axis is therefore time related. Ligamentous injuries, however, can take longer to heal. An isolated ligamentous injury without any osseous damage to the unit of motion with or without facet joint malalignment may be an indication for surgery. The aim of the management of the biomechanical instability (surgically or with APCM) is therefore the "containment" of the instability until tissue healing occurs and stability is restored. Biomechanical instability is safely contained in recumbence for 4-6 weeks followed by bracing for a further six weeks during mobilisation and active rehabilitation.

Wagih El Masri demonstrated that with APCM almost all biomechanically unstable injuries become biomechanically stable and pain free. APCM respects the segmental architecture of the vertebral axis by achieving the shortest fusion that preserves the flexibility of the injured motion segments of the spine and enables the patient to achieve an almost normal range of painless movement Containment of the biomechanical instability by surgical means is likely to be effective as long as the instrumentation remains sound. Failure of instrumentation before biomechanical stability is restored is likely to increase the risk of cord damage during mobilisation and/or rehabilitation. It must be appreciated that the forces and vectors of forces on the spinal axis are different in the neurologically intact patient than in the neurologically impaired patient. The neurologically impaired patient needs to twist and bend (often simultaneously) in a wheelchair during rehabilitation and to achieve independence in dressing, undressing, transferring from a level or from floor to chair, tying shoe laces etc. The neurologically impaired patient is more at risk of instrumentation failure during the early stages of participation in an active programme of structured rehabilitation than the neurologically intact patient who hardly requires any rehabilitation. The degree of kyphotic deformity may be lower following surgical stabilisation than following APCM. The greatest majority of these residual kyphotic deformities in patients treated with APCM are however painless and the discrepancy between deformity and pain has been known for some time. ${ }^{24}$ It must be appreciated that for wheelchair-dependent patients a painless kyphotic deformity is likely to enhance independence and is certainly, much more preferable to a stiff straight neck or back following surgical instrumentation with or without fusion.

\section{Traumatic canal encroachment}

Some of the first case reports to suggest that traumatic canal encroachment as demonstrated by computerised tomography does not correlate with the degree of neurological impairment, does not prevent neurological recovery and does not result in neurological deterioration were published by El Masri et al., in 1992. The same conclusions were made by reviewing the outcome of conservative treatment of 50 consecutive patients with between $10 \%$ and $90 \%$ canal encroachment in Frankel C, D and E groups; patients in Frankel $C$ and $D$ group recovered ambulation and none of the patients deteriorated neurologically or otherwise Other groups have since published similar findings.

The majority of patients with TSCI are currently surgically decompressed and/or stabilised without the rigours of adequate research methodology or demonstration of equality or superiority of neurological and/or other outcomes over APCM. This can be contrasted with practice in our orthopaedic institution (with five dedicated spinal surgeons) where the great majority of patients with TSCI are treated conservatively. A recent study revealed that only $2.8 \%$ of patients (who are transferred without any spinal surgical management to our institution) are surgically managed. ${ }^{31}$

The debate over the effects of the timing of surgical spinal decompression after TSCI has remained unresolved for over a century. The Surgical Timing in the Acute Spinal Cord Injury Study (STASCIS) by Fehlings et al. reported that surgical decompression prior to $24 \mathrm{~h}$ is safe and improved neurologic outcome more than late decompression (after $24 \mathrm{~h}$ ), with improvement was defined as recovery of at least two AIS grades at 6 months follow-up. ${ }^{32}$

The STASCIS study drew criticism for poor sample size calculation, lack of significant difference for one ASIA Impairment Scale (AIS) group, a technically questionable analytical approach and an odds ratio in the early surgery group not meeting statistical significance. ${ }^{33}$ In 2012, Van Middendorp performed a systematic review and quality-adjusted meta-analysis of studies (1966 to August 2012) evaluating the effects of the timing of spinal surgery after TSCI concluding that the published evidence lacks robustness as a result of different sources of heterogeneity within and between original studies. ${ }^{33}$

\section{Case report (Figs. 2-5) - a natural history observation study of lumbar spinal injuries with canal encroachment}

A 37-year-old lady sustained a fall from a height of about $5 \mathrm{~m}$ in Greece. She was flown to the UK the following day and admitted to

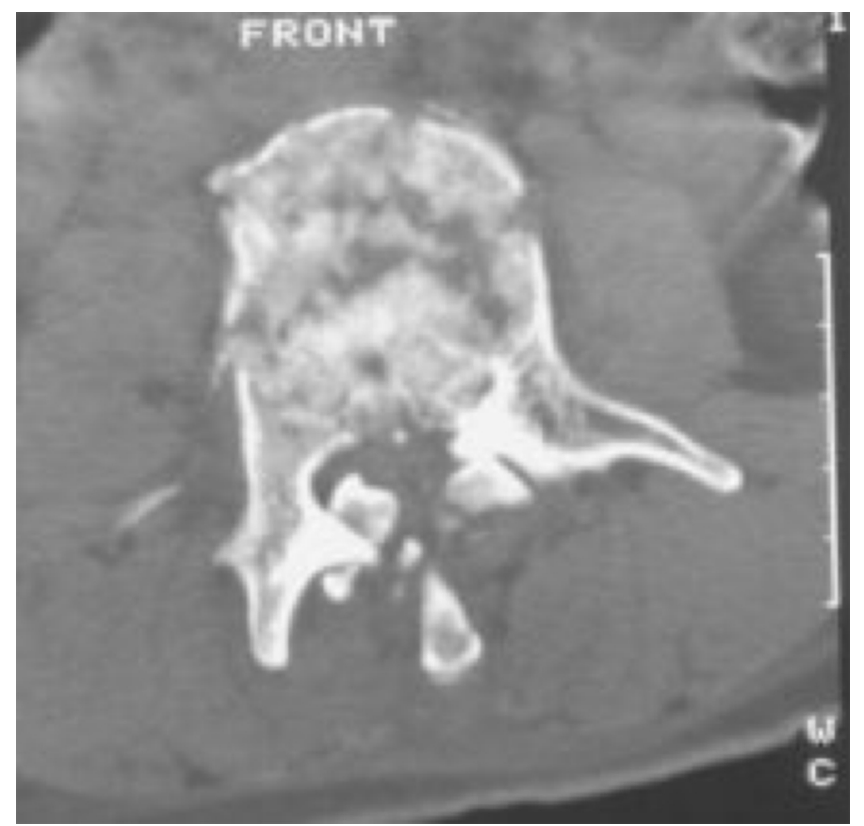

Fig. 2. Axial CT confirming the burst fracture, malalignment and canal encroachment. 


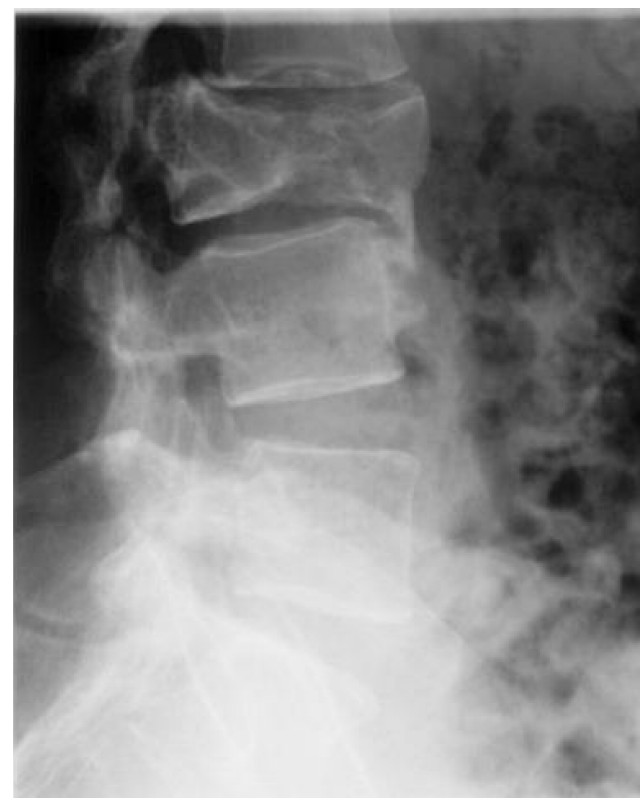

Fig. 3. Lateral X-ray confirming healed fracture and malalignment.

the MCSI with profound Frankel D paraparesis, numbness in both legs and a catheter in her bladder. Her motor score in the lower limb was 75/100. Radiological examination demonstrated an L3 burst fracture and fracture dislocation of L2/L3. She underwent APCM of her fracture and multisystem malfunction with 6 weeks of bed rest followed by 6 weeks in a plaster cast. Attempts at postural reduction and realignment failed. The fracture dislocation was not reduced and the alignment was not restored. She was discharged home eight weeks following the accident having regained full motor power, normal sensation, control of bladder and bowel functions and was able to walk without arm support or lower limb orthosis. 26 years after the accident she continues to enjoy a pain free full range of movement full control over her bladder and bowels, the ability to walk unsupported and run, and maintains full independence in all activities of daily living including the ability to drive a manual car and swim regularly.

\section{Mobilisation}

Early mobilisation is advantageous to neurologically intact patients with stable fractures or indeed unstable fractures following surgical stabilisation. These patients can ambulate and be safely discharged to their own homes soon after surgery. Based on a cohort study of 60 adult patients presenting sequentially over 15 years with thoracic and/or lumbar vertebral burst fractures, but without neurological deficit, Jaffray et al. have demonstrated that in the neurologically intact patients, spinal canal encroachment had no influence on successful functional recovery and pain was not a significant problem for any patient, irrespective of the degree of kyphosis and no patient had self-perception of clinical deformity. ${ }^{39}$ This is similar to the outcomes demonstrated in patients with TSCI by El Masri since the early 1990s. ${ }^{24}$

SCI leads to dysfunctional physiology of various internal organs including spinal cord itself. This is predominantly because of the altered/loss of balance between the opposite but complementary effects of sympathetic and parasympathetic systems in addition to the dysfunctional somato-sensory and motor components of the nervous system. Physiological instability of the spinal cord was well described by El Masri. Impaired sympathetic control and excess parasympathetic activity plays a crucial role in physiological instability of the spinal cord and secondary injury. It is not uncommon to see patients with neurological deterioration following early and/or sudden mobilisation and this is can be explained by secondary injury.

The majority of patients with incomplete SCI show signs of, or start to recover neurologically, during the first six weeks period of immobilisation. All these patients are at risk of developing postural hypotension and neurological deterioration (Tables 1 and 2).

At Midland Centre for Spinal Injuries a process of close monitoring of blood pressure and neurological status throughout varying degrees of inclination, on a tilt table, prior to mobilisation of the all incomplete SCI patients known as the Tilt Table Study (TTS) is routinely carried out prior to mobilisation. From a retrospective review of 263 tilt table studies carried out at the MCSI since 1990, 167 (63.5\%) TTS were completed without complication, 71(27.0\%) were stopped due to hypotension, 5 (1.9\%) stopped due to hypertension (autonomic dysregulation). 20 (7.6\%) TTS were stopped due to neurological deterioration being detected (Table 3 ).
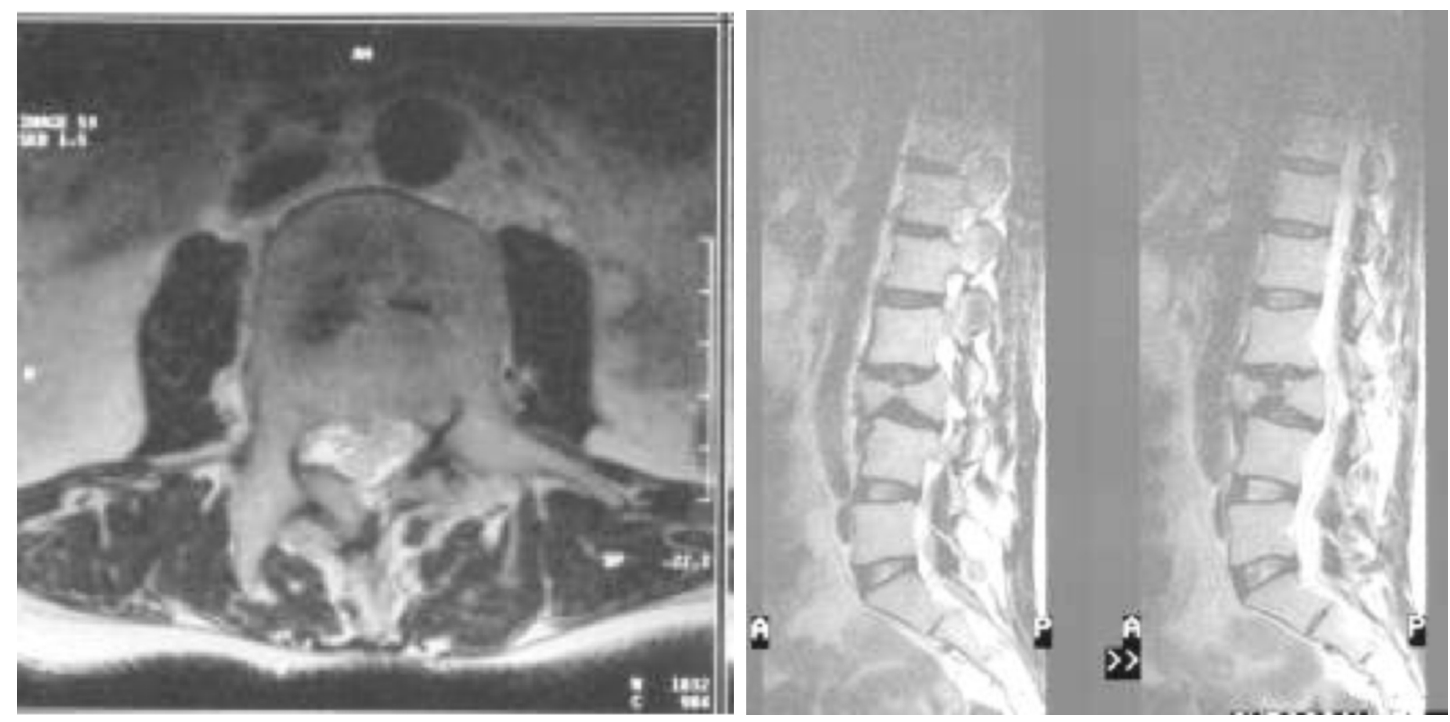

Fig. 4. (a) and (b) Axial and Sagittal section of MRI, 4 years later confirms ongoing thecal and cauda equina compression. 

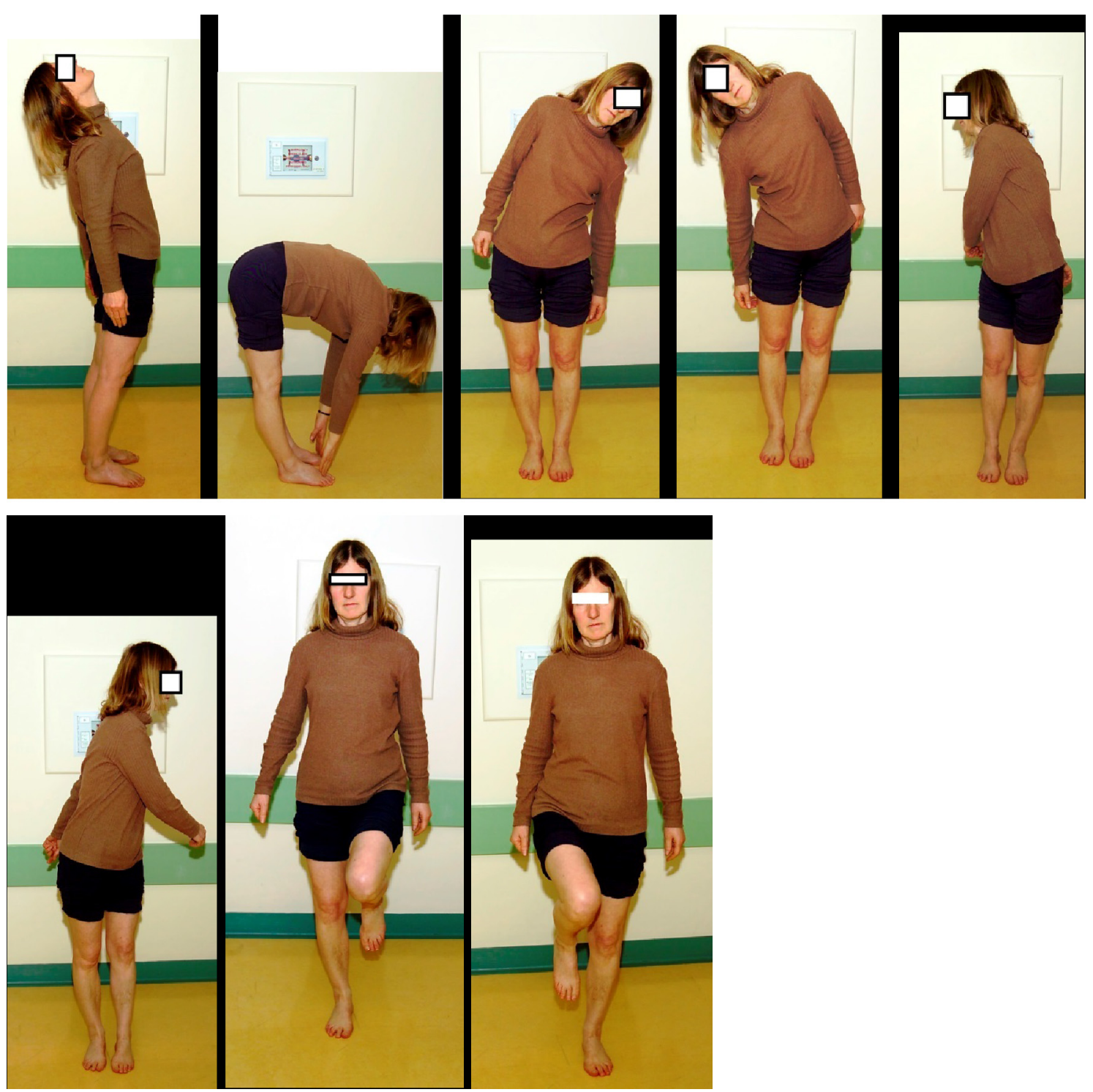

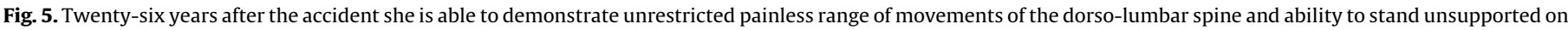
one leg at a time.

The Tilt Table Studies do enable the patients at risk to build confidence in the multi-disciplinary team, reducing their anxiety and allowing their full participation in the early stages of their rehabilitation. Knowing how these patients respond to the challenge of verticalisation allows an individualisation of the process of their mobilisation. This in turn ensures efficient time management and good planning of rehabilitation. TTS enable the team to identify patients who are likely to deteriorate neurologically during the early stages of mobilisation.

Patients with paralysis, general physiological impairment and multisystem malfunction do not benefit from early mobilisation, which may in fact be deleterious in more than one way to the patient. Early mobilisation during the stage of spinal shock results in profound hypotension which can reduce cord perfusion.

Table 1

The number of acute admissions at the MCSI between 2007/2008 and 2014/2015

\begin{tabular}{|c|c|c|c|c|c|}
\hline & Total No. of patients & Age 65 \& Over (\%) & Age 60-64 (\%) & Age $50-59(\%)$ & Under 50 (\%) \\
\hline Prior 1965 & 41 & 0 & 0 & 5 & 95 \\
\hline $1965-1974$ & 238 & 1 & 1 & 4 & 92 \\
\hline $1975-1984$ & 441 & 1 & 2 & 8 & 86 \\
\hline 1985-1994 & 1276 & 7 & 5 & 12 & 64 \\
\hline 1995-2004 & 2274 & 9 & 7 & 17 & 51 \\
\hline 2004 to date & 1189 & 11 & 8 & 21 & 41 \\
\hline
\end{tabular}

There are 1690 records that do not have a readmission date so these have been excluded.

If a patient has attended more than once in a decade these figures have been included. 
Table 2

Changes in the age at time of Spinal Cord Injury - MCSI data.

\begin{tabular}{lll}
\hline & Traumatic & Non-traumatic \\
\hline $2007 / 08$ & 86 & 11 \\
$2008 / 09$ & 61 & 16 \\
$2009 / 10$ & 72 & 17 \\
$2010 / 11$ & 63 & 16 \\
$2011 / 12$ & 105 & 24 \\
$2012 / 13$ & 98 & 13 \\
$2013 / 14$ & 111 & 22 \\
$2014 / 15$ & 110 & 20 \\
\hline
\end{tabular}

Individuals with spinal cord injury exhibit reduced lung volumes and flow rates as a result of respiratory muscle weakness. These features have been investigated in relation to the combined effects of injury level and posture. Values of forced vital capacity and forced expiratory volume in one second (FEV1) were repeatedly and consistently shown to be larger in recumbence compared to the seated posture. ${ }^{40-43}$ Early mobilisation of patients with spinal neural tissue injury is associated with a reduction of vital capacity and a potential drop of oxygen saturation. During the stage of spinal shock, early mobilisation of the tetraplegic and high paraplegic results in further marked impairment in the patient's ability to cough. It is more difficult to implement postural drainage and get rid of bronchial secretions with assisted coughing against gravity while the patient is sitting upright than when the patient is recumbent. Individually, or in combination, these respiratory and vascular pathophysiological mechanisms can potentially cause further impairment of cord functions.

Appropriate posture and timing of mobilisation play an important role in the prevention of deterioration of physiological stability of the spinal cord and neurological function. Furthermore, it is more difficult to carry out intermittent catheterisation, bowel evacuation or manage episodes of urinary or bowel incontinence of a patient of a wheelchair than in recumbency. During the stage of spinal shock, skin perfusion is markedly diminished and the skin over bony prominences is at its peak of vulnerability. While in recumbency the weight of the patient is spread across the body and all the bony prominences while in the sitting position, most of the weight of the patient is usually concentrated over the skin of the ischial tuberosity and the sacrum, increasing the risk of pressure sores over these bony prominences.

\section{Natural history}

Fewer than $10 \%$ of patients initially with clinically complete spinal cord injuries (Frankel grade A, "FA") improve to make a significant recovery to ambulate with APCM. Many more however, recover cord functions in one to four myotomal distributions below the level of the injury or improve to FB and FC. ${ }^{17}$ Although

Table 3

Neurological deterioration seen in incomplete SCI patients during the Tilt Table Studies.

\begin{tabular}{|c|c|c|c|c|}
\hline Neurological level & Commencing blood pressure (BP) & Angle of inclination the change occurred & $\mathrm{BP}$ at time of change & Neurological change \\
\hline C5 & $114 / 70$ & $20^{\circ}$ & $122 / 79$ & $\begin{array}{l}\text { C5 sensation } \\
\text { Normal (2) to altered (B) }\end{array}$ \\
\hline C5 & $150 / 81$ & $30^{\circ}$ & $89 / 58$ & Triceps MRC Grade 3 to Grade 2 \\
\hline C5 & $102 / 66$ & $20^{\circ}$ & $95 / 72$ & $\begin{array}{l}\text { Grip strength } 25.5-21.6 \mathrm{lbs} \\
\text { T1 sensation } \\
\text { Normal }(2) \text { to altered }(\mathrm{A})\end{array}$ \\
\hline C6 & $122 / 76$ & $40^{\circ}$ & $115 / 81$ & Grip strength 9-6 lbs \\
\hline C3 & $153 / 103$ & $30^{\circ}$ & $131 / 89$ & $\begin{array}{l}\text { Biceps Grade } 3 \text { to } 3- \\
\text { C5 sensation altered } A \text { to } B\end{array}$ \\
\hline C6 & $99 / 59$ & $40^{\circ}$ & $82 / 56$ & $\begin{array}{l}\text { Quadriceps Grade } 2+\text { to } 2- \\
\text { C6 sensation normal to altered }\end{array}$ \\
\hline C6 & $169 / 104$ & $50^{\circ}$ & $121 / 83$ & Quadriceps $4-$ to 3+ \\
\hline C3 & $106 / 72$ & $50^{\circ}$ & $100 / 56$ & $\begin{array}{l}\text { (Tested with a myometer) } \\
\text { Wrist extensor 29-21 } \\
\text { Quadriceps } 41-30\end{array}$ \\
\hline C5 & $107 / 67$ & $30^{\circ}$ & $102 / 75$ & $\begin{array}{l}\text { Pincer grip reduced } 12.2-7.2 \mathrm{lbs} \text { and } \\
\text { Dorsiflexors Grade } 5 \text { to Grade } 4\end{array}$ \\
\hline C7 & $112 / 65$ & $40^{\circ}$ & $90 / 56$ & Grip strength reduced from 17 to $7 \mathrm{lbs}$ \\
\hline C5 & $135 / 79$ & $50^{\circ}$ & $112 / 80$ & $\begin{array}{l}\text { T1 sensation normal (2) to altered (A) } \\
\text { Grip strength reduced from } 15 \text { to } 12 \mathrm{lbs}\end{array}$ \\
\hline
\end{tabular}

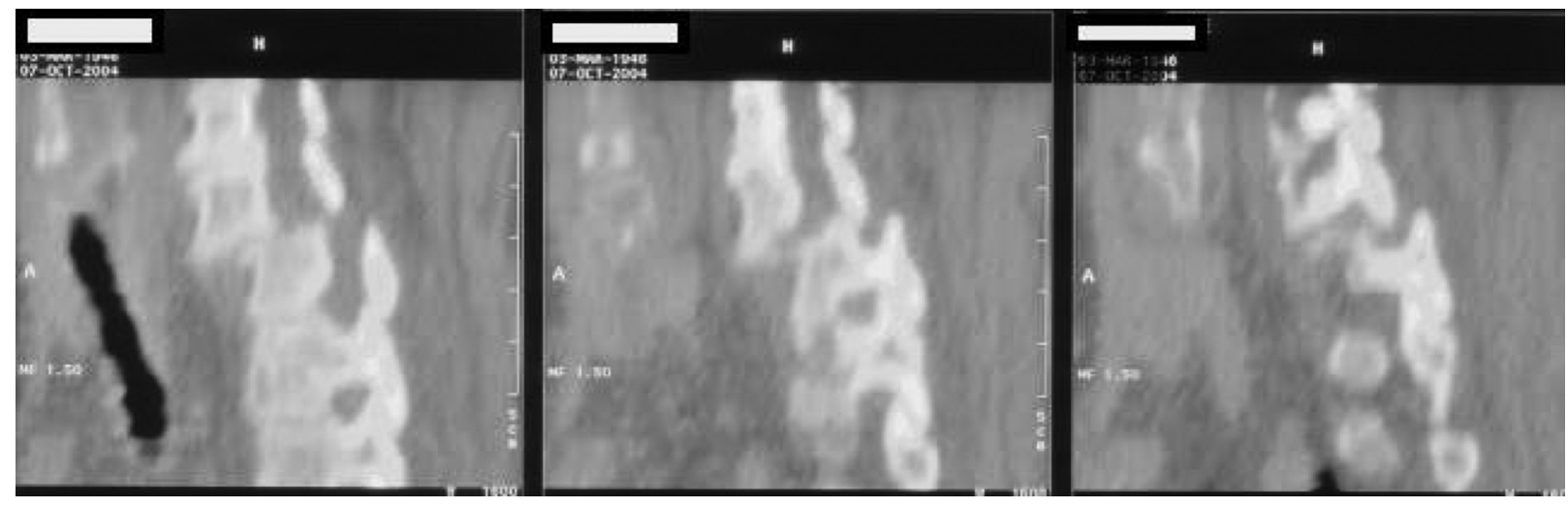

Fig. 6. Lateral CT scan image confirming the fracture, malalignment and the canal encroachment at C6-C7 level. 


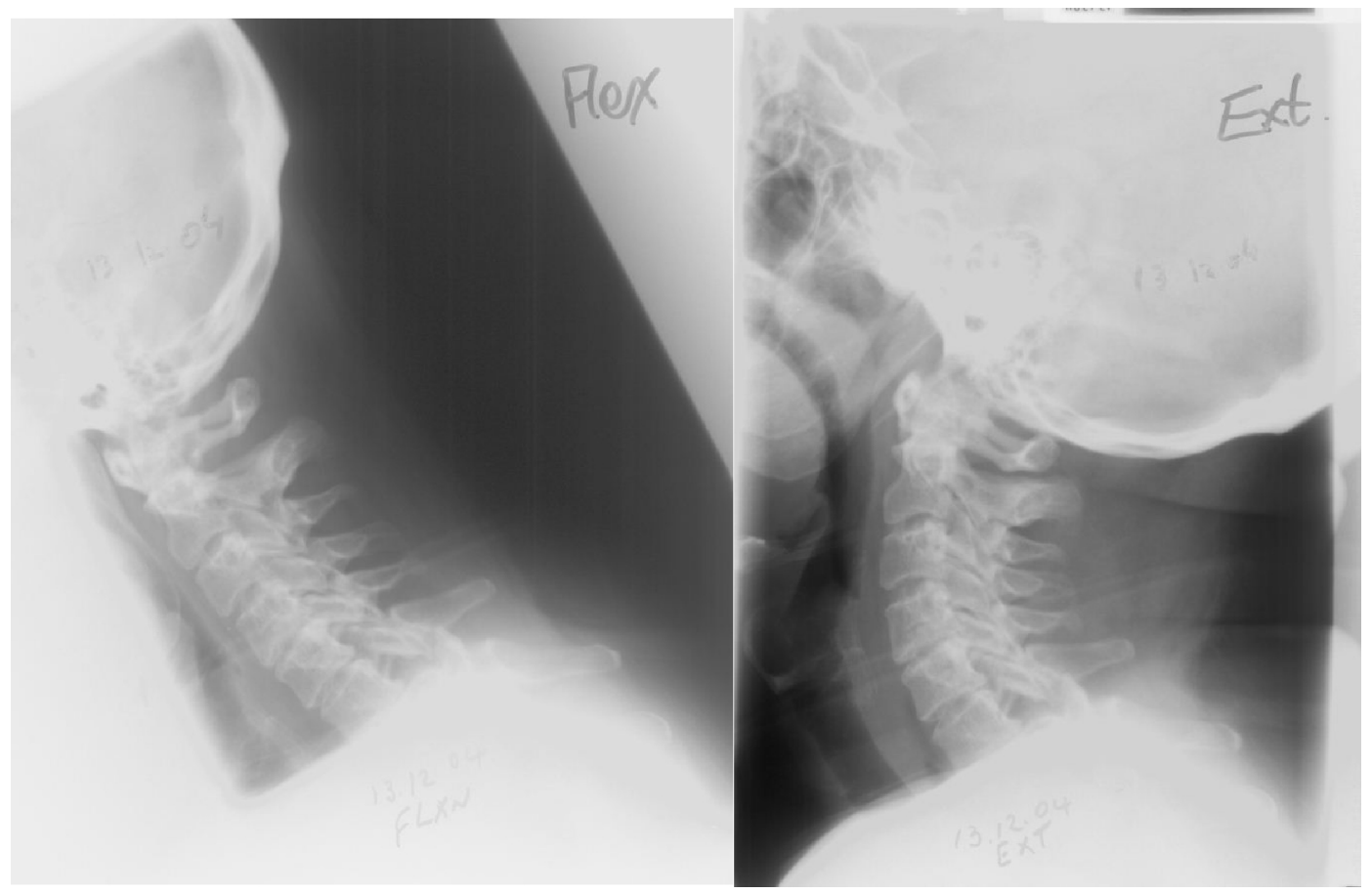

Fig. 7. (a) and (b) Lateral X-ray (flexion-extension dynamic radiograph) confirming stability, healing fracture dislocation and malalignment at C6-C7 level.

since the 1980s anterior surgical decompression and arthrodesis have become increasingly established practice, based on suggestions that surgery resulted in motor zonal improvement; to date there is no evidence that surgery provides added value. A series of 53 consecutive patients with complete traumatic tetraplegia, admitted to one centre within two days of injury, demonstrated that similar results can be achieved without surgical decompression or arthrodesis. ${ }^{8}$ Patients with incomplete cord injuries make significant neurological recovery irrespective of the degree of canal stenosis, canal encroachment, misalignment or cord compression provided both the biomechanical Instability of the spinal column and the Physiological Instability of the spinal cord are well contained by APCM. ${ }^{5,7,15,21}$

\section{Case report (Figs. 6-9) - a natural history observation of cervical spinal injuries with cord compression}

A 58-year-old lady sustained a fall out of bed, resulting in profound Frankel $\mathrm{C}$ tetraparesis from a C6/C7 level. On admission to the MCSI 15 days following injury, she had an indwelling catheter and her motor score was 47/100. MRI scan confirmed C6/ C7 bilateral facet dislocations with malalignment and canal encroachment. High cord signal was noted at $\mathrm{C6} / \mathrm{C7}$. The dislocation was not reduced and the alignment was not restored. Her spinal injury and the multisystem malfunction were managed with APCM for a period of nine weeks in bed followed by mobilisation in a soft collar. She was discharged home 17 weeks following the accident having recovered full motor power and sphincter control. She was able to walk indoors without arm support or lower limb orthosis. She held her husband's hand while walking outdoors for a period of time. Despite osteoarthritic changes in her hips, lower back and neck, she continues to enjoy a pain-free good range of movement. She continues to be independent in all aspects of daily living 12 years following the accident with unrelieved cord compression and without clinical signs or symptoms of post traumatic syringomyelia. Because of her age and arthritic hips, she requires one stick for support during ambulation. She continues to be monitored neurologically.

\section{Conclusions}

To date there is no evidence to suggest that the surgical decompression or stabilisation of the spinal cord injured patient is advantageous. Until credible evidence is available to demonstrate superiority of outcome with early surgery compared to early active treatment of the injured spine, patients should be encouraged to

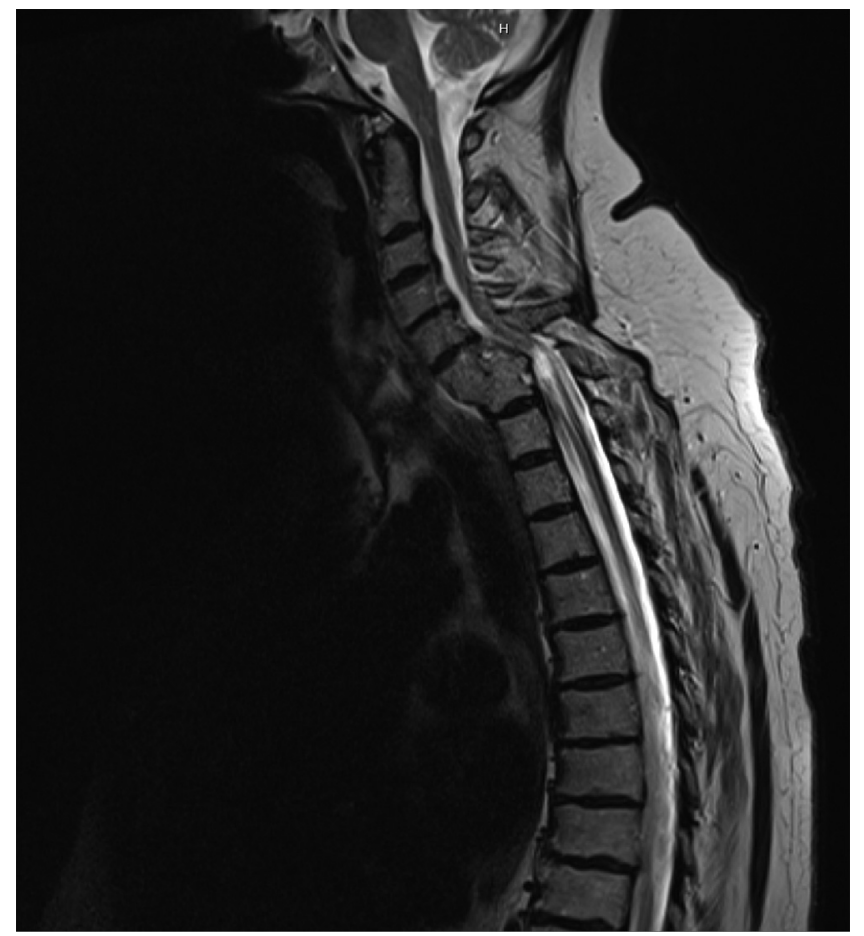

Fig. 8. Sagittal section of T2 weighted MRI thirteen years later confirms, ongoing cord compression old C6-C7 fracture dislocation with anterior fusion in situ. Posttraumatic cystic change within the cord is noted at this level producing a fusiform expanded cord contour as well as myelomalacia proximally and distally. 

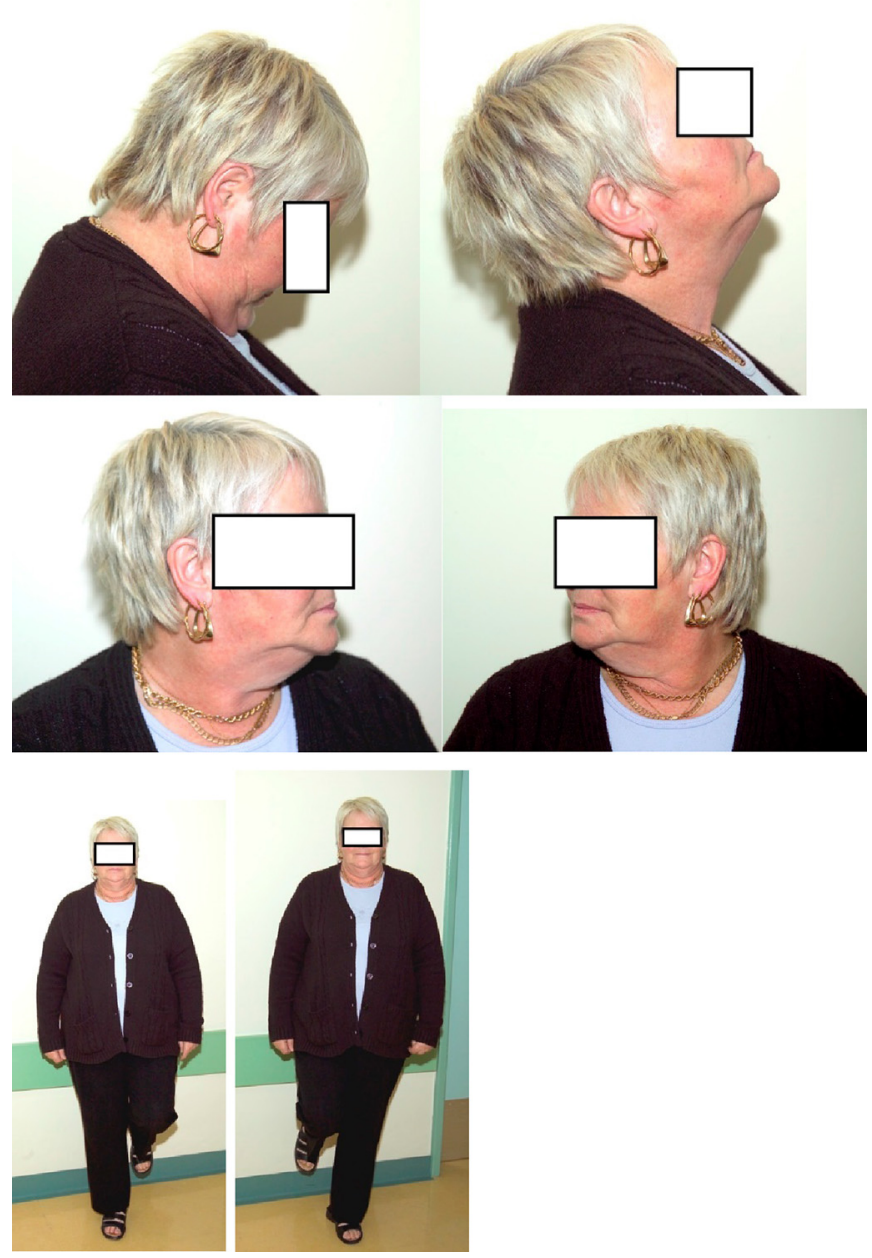

Fig. 9. Thirteen years after the accident she is able to demonstrate unrestricted painless range of movements of the cervical spine and ability to stand unsupported on one leg at a time.

manage an informed choice. The majority of patients with intact neurology but a biomechanically unstable spine benefit from surgical stabilisation of the spine. The established simultaneous active physiological conservative management of the spinal cord injury and all its effects continues to demonstrate excellent results irrespective of the mechanism of injury, radiological appearances and degree of biomechanical instability or presence of cord compression. Although surgery is likely to be necessary for a small number of patients with damage of the cord or cauda equina, the surgical management of the spine is best provided in a dedicated spinal centres where the knowledge, expertise, skills and necessary infrastructure are available to simultaneously adequately manage the multisystem impairment, malfunction as well as the nonmedical effects of paralysis. The ultimate goals of $\mathrm{SCI}$ management should be to ensure maximum neurological recovery, independence, a pain free flexible spine, safe functioning of the various systems of the body with minimal or no inconvenience to patients and prevention of complications.

\section{Conflicts of interest}

The authors have none to declare.

\section{Uncited references}

$$
1-4,6,9-16,18,20,22,23,34-38,44-47 .
$$

\section{Acknowledgements}

The authors would like to thank all the patients, Prof. Wagih El Masri and physiotherapy staff for their guidance and/or support without which the publication of this article would not have been possible.

\section{References}

1. Cooper AP, Cline H. Of wounds of the scalp. Lancet. 1824;1:393-398.

2. Bell C. Observations on Injuries of the Spine and Thigh Bone. Vol. 73. London: Thomas Tegg.; 1824: 3-31.

3. Guttmann L. Spinal Cord Injuries: Comprehensive Management and Research. 2nd ed. Oxford: Blackwell; 1976

4. Davis AG. Fractures of the spine. J Bone Joint Surg. 1929;11:133.

5. Rogers WA. Surg Gyn Obstet. 1930;50:101.

6. Bohler L. The Treatment of Fractures. Bristol: Wright \& Son; 1935.

7. Watson-Jones. Sir Reg. J Bone Joint Surg. 1931;13:383.

8. Watson-Jones. Sir Reg. J Bone Joint Surg. 1934;16:30.

9. Dunlop J, Parker CH. J Bone and Joint Surg. 1933;15(if3 (January)).

10. Nissen K. Proc R Soc Med. 1942;35:707.

11. Guttmann L. Med Times N Y. 1945;73:318.

12. Guttmann L. Nurs Times. 1946;42:798.

13. Watson-Jones R. Fractures and Joint Injuries. Fourth ed.; 1955.

14. Holdsworth FW, Hardy AG. J Bone Joint Surg. 1953;3SB:540.

15. Frankel HL, Hancock DO, Hyslop G, et al. The value of postural reduction in initial management of closed injuries of the spine with paraplegia and tetraplegia. Paraplegia. 1969-70;7:179-192.

16. El Masri W. Management of traumatic spinal cord injuries: current standard of care revisited. ACNR. 2010;10(March/April):37-40.

17. Katoh S, El Masry WS. Motor recovery of patients presenting with motor paralysis and sensory sparing following cervical spinal cord injuries. Paraplegia. 1995;33:506-509.

18. Katoh S, El Masri(y) WS. Neurological recovery after conservative treatment of cervical cord injuries. J Bone Joint Surg Br. 1994;76B:225-228.

19. El Masry WS. Editorial - physiological instability of the injured spinal cord. Paraplegia. 1993;31:273-275.

20. Folman Y, El Masri WS. Spinal cord injury: prognostic indicators. Injury. 1989;20:92-93.

21. Katoh S, El Masry WS, Jaffray D, et al. Neurologic outcome in conservatively treated patients with incomplete closed traumatic cervical spinal cord injuries. Spine. 1996;21:2346-2351.

22. Kwon BK, Curt A, Belanger LM, et al. Intrathecal pressure monitoring and cerebrospinal fluid drainage in acute spinal cord injury: a prospective randomized trial. $J$ Neurosurg Spine. 2009;10:181-193.

23. El Masri(y) WS, Kumar N. Traumatic spinal cord injuries. Lancet. 2011;377(9770 (March)):972-974.

24. El Masry WS. Traumatic spinal cord injury: the relationship between pathology and clinical implications. Trauma. 2006;8:29-46.

25. El Masri WS, Jaffray D. Recent developments in the management of injuries of the cervical spine. Spinal cord trauma. Handbook of Clinical Neurology; rev series; 17. Vol. 61. Elsevier Science; 1992[Chapter 3].

26. El Masri WS, Meerkotter DV. Early Decompression of the Spinal Cord Following Injury: Arguments for and Against. Spinal Cord Dysfunction Vol II: Intervention and Treatment. Oxford University Press; 1992. 7-2.

27. El Masri (y) WS, Katoh S, Khan A. Reflections on the neurological significance of bony canal encroachment following traumatic injury of the spine in patients with Frankel C, D and E presentation. J Neurotrauma. 1995;10(suppl):70.

28. Limb D, Shaw DL, Dixon RA. Neurological injury in thoracolumbar burst fractures.J Bone Joint Surg Br. 1995;77B:774-777.

29. Rosenberg N, Lenger R, Weisz I, Stein H. Neurological deficit in a consecutive series of vertebral fractures patients with bony fragments within the spinal canal. Spinal Cord. 1996;35:92-95.

30. Boerger TO, Limb D, Dickson RA. Does canal clearance affect neurological outcome after thoracolumbar burst fractures. J Bone Joint Surg Br. 2000;82B:629-635.

31. Patil S, Myint M, Kumar N. Indications and outcomes of surgical management of acute traumatic spinal cord injury. Eur Spine J. 2013;22(1 suppl (March)):7/27.

32. Fehlings MG, Vaccaro A, Wilson JR, et al. Early versus delayed decompression for traumatic cervical spinal cord injury: results of the Surgical Timing in Acute Spinal Cord Injury Study (STASCIS). PLoS ONE. 2012;7(2):e32037.11.

33. Van Middendorp JJ, Hosman AJ, Doi SA. The effects of the timing of spinal surgery after traumatic spinal cord injury: a systematic review and meta-analysis. $J$ Neurotrauma. 2013;30(21 (November)):1781-1794.

34. Brennan P. STASCIS Results on Timing in Spinal Injury Surgeons News. The Royal College of Surgeons of Edinburgh; 2012, June:67.

35. Dolan EJ, Tator CH, Endrenyi L. The value of decompression for acute experimental spinal cord compression injury. J Neurosurg. 1980;53:749-755.

36. Guho A, Tutor CH, Endemic L, Piper I. Decompression of the spinal cord improves recovery after acute experimental spinal cord compression injury. Paraplegia. 1987;25:324-339.

37. Tator CH, Duncan EG, Edmonds VE, Lapczac LI, Andrews DF. Comparison of surgical and conservative management of 208 patients with acute spinal cord injury. Can J Neurol Sci. 1987;14(1):60-69. 
38. Courtine G, Bunge MB, Fawcett JW, et al. Can experiments in nonhuman primates expedite the translation of treatments for spinal cord injury in humans? Nat Med. 2007; $13: 561-566$.

39. Ibrahim A, Li Y, Li D, Raisman G, El Masry WS. Olfactory ensheathing cells: ripples of an oncoming tide? Lancet Neurol. 2006;5:453-457.

40. Morgan MDL, Silver JR, Williams SJ. The respiratory system of the spinal cord patient. In: Bloch RF, Basbaum M, eds. In: Management of Spinal Cord Injury. Baltimore: Williams and Wilkins; 1986:78-117.

41. Cameron GS, Scott JW, Jousse AT, Botterell EH. Diaphragmatic Respiration in the Quadriplegic Patient and the Effect of Position on His Vital Capacity. Ann Surg. 1955;141(4 (April)):451-456.

42. Baydur A, Adkins RH, Milic-Emili J. Lung mechanics in individuals with spinal cord injury: effects of injury level and posture. J Appl Physiol. 2001;90(2 (February)):405-411.
43. Alvisi V, Marangoni E, Zannoli S, et al. Pulmonary function and expiratory flow limitation in acute cervical spinal cord injury. Arch Phys Med Rehabil. 2012;93(11 (November)):1950-1956.

44. Carvell JE, Grundy DJ. Complications of spinal surgery in acute spinal cord injury. Paraplegia. 1994:2:389-395.

45. Aung TS, El Masry WS. Audit of a British centre for spinal injury. Spinal Cord 1997; $35: 147-150$.

46. Bagnall AM, Jones L, Duffy S, Riemsma RP. Spinal fixation surgery for acute traumatic spinal cord injury. Cochrane Database Syst Rev. 2008;(1 (January)):CD004725.

47. Jaffray DC, Eisenstein SM, Balain B, Trivedi JM, Newton Ede M. Early mobilisation of thoracolumbar burst fractures without neurology: a natural history observation. Bone Joint J. 2016;98-B(1 (January)):97-101. 